\title{
Natural optical anisotropy of h-BN: Highest giant birefringence in a bulk crystal through the mid-infrared to ultraviolet range
}

\author{
A. Segura, ${ }^{1}$ L. Artús, ${ }^{2}$ R. Cuscó, ${ }^{2}$ T. Taniguchi,${ }^{3}$ G. Cassabois,${ }^{4}$ and B. Gil ${ }^{4, *}$ \\ ${ }^{1}$ Departamento de Física Aplicada-ICMUV, Malta-Consolider Team, Universitat de València, Burjassot, Spain \\ ${ }^{2}$ Institut Jaume Almera (ICTJA-CSIC), Consejo Superior de Investigaciones Científicas, 08028 Barcelona, Spain \\ ${ }^{3}$ National Institute for Materials Science, 1-1 Namiki, Tsukuba, 305-0044, Japan. \\ ${ }^{4}$ Laboratoire Charles Coulomb (L2C), UMR 5221 CNRS-Université de Montpellier, F-34095, Montpellier, France
}

(Received 29 November 2017; published 6 February 2018)

\begin{abstract}
The giant birefringence of layered h-BN was demonstrated by analyzing the interference patterns in reflectance and transmittance measurements in the mid-infrared to the deep ultraviolet energy range. The refractive index for polarization perpendicular to the $c$ axis is much higher than the refractive index for polarization parallel to the $c$ axis, and it displays a strong increase in the ultraviolet range that is attributed to the huge excitonic effects arising from the unique electronic structure of h-BN. Thus, h-BN is shown to exhibit a giant negative birefringence that ranges from -0.7 in the visible to -2 in the deep ultraviolet close to the band gap. The electronic dielectric constants for polarization perpendicular and parallel to the $c$ axis were determined to be $\varepsilon_{\infty}^{\perp}=4.95$ and $\varepsilon_{\infty}^{\|}=2.86$, respectively. The anisotropy we find in high-quality h-BN is significantly larger than proposed in previous experimental studies but in excellent agreement with ab initio calculations.
\end{abstract}

DOI: 10.1103/PhysRevMaterials.2.024001

\section{INTRODUCTION}

Hexagonal boron nitride (h-BN) is a van der Waals crystal that has been intensively studied over the last few years for the high interest of its numerous potential applications. The wide band gap of this layered material $(\sim 6 \mathrm{eV})$ [1] and its high emission efficiency [2] make h-BN a material of special interest for deep ultraviolet photonic device applications [3-5]. In spite of the fact that $a b$ initio band structure calculations long predicted an indirect band gap for h-BN [6,7], only recently the experimental demonstration of the indirect nature of the h-BN band gap has been provided [8]. Because of the similarity between the in-plane lattice constant of h-BN and graphene, h-BN is ideally suited to form van der Waals stacked heterostructures. The exceptional crystalline quality of such structures has opened up new avenues for exploring novel physics and potential applications of stacked two-dimensional materials $[9,10]$. By encapsulating graphene in h-BN layers, graphene devices exhibiting extremely high electron mobility at room temperature were demonstrated [11]. Atomically thin graphene-boron nitride lateral heterostructures have been realized that may enable the development of atomically thin integrated circuits [12]. Besides its prominent role in advanced optoelectronics, h-BN has also emerged as a material with unique properties for solid-state neutron detector applications [13].

In spite of the huge amount of research recently reported on $\mathrm{h}-\mathrm{BN}$, the knowledge of its optical properties is rather limited, possibly because of the difficulties in growing sizable samples with high crystal quality. Spectroscopic ellipsometry studies on amorphous BN films deposited by rf magnetron sputtering

\footnotetext{
*On leave at the Ioffe Physical-Technical Institute, Polytekhnicheskaya 26, St. Petersburg, 194021, Russia.
}

consisting of mixed cubic-BN and h-BN phases reported the dielectric function in a wide energy range [14]. Values of the macroscopic dielectric constant along the $c$ axis $\left(\varepsilon^{\|}\right)$and perpendicular to the $c$ axis $\left(\varepsilon^{\perp}\right)$ were reported in infrared reflectivity studies on $\mathrm{h}-\mathrm{BN}$ polycrystalline samples grown by pyrolytic methods [15]. Ab initio calculations indicate that the layered structure of h-BN induces a remarkably strong optical anisotropy $[7,16]$, which was not confirmed by the experimental work of Geick et al. [15], probably because the polycrystalline nature of the samples precluded an accurate determination of pure $\varepsilon^{\|}$and $\varepsilon^{\perp}$ values. To our knowledge, the disparity between theoretical and experimental values of the dielectric function anisotropy has not been elucidated yet.

In recent times, a great deal of effort has been devoted to develop semiconductor nanostructures exhibiting large artificial birefringence for applications in quantum optics [17], polarization sensitive optoelectronics [18], and microphotonic polarization converters applications [19]. Birefringence values as high as $\Delta n=0.8$ at $633 \mathrm{~nm}$ have been reported in ensembles of $\mathrm{GaP}$ nanowires [20], which are much larger than the birefringence of the $\mathrm{YVO}_{4}$ crystal $(0.21$ at $633 \mathrm{~nm})$ widely used in laser devices and optical components [21].

While the structural anisotropy intrinsic to all van der Waals crystals is presumed to produce a strong optical birefringence, detailed experimental studies of birefringence in these materials are lacking. Even though on account of its layered structure the h-BN crystal is expected to be highly optically anisotropic, and indeed it has emerged as a natural hyperbolic crystal suitable for supporting phonon polariton modes [22], birefringence of $\mathrm{h}-\mathrm{BN}$ has not received much attention despite the vast variety of technological applications of this material. The deceptively low value of dielectric constant anisotropy reported in Ref. [15] may have contributed to dim the interest of h-BN as a birefringent crystal. 
Here we report a determination of the refractive index anisotropy of h-BN in a wide energy range from mid-infrared (MIR) to ultraviolet (UV) from polarized transmittance measurements. The high-frequency dielectric constant was evaluated from fits to reflectivity measurements in the MIR. The ordinary refractive index as a function of wave number $n_{\perp}(\sigma)$ was then obtained from the transmittance interference fringe pattern measured under normal incidence on the $c$ face. The small thickness of the high quality h-BN samples and the uneven nature of the lateral faces resulting from their layered structure preclude polarized transmittance measurements with the electric field parallel to the $c$ axis. Therefore, the extraordinary refractive index dispersion $n_{\|}(\sigma)$ was determined from the analysis of transmittance measurements on the $c$ face at various incidence angles with polarization in the incidence plane and perpendicular to it.

\section{EXPERIMENT}

High quality h-BN single crystals were synthesized at 4.5 $\mathrm{GPa}$ and $1500^{\circ} \mathrm{C}$ using barium boron nitride as a solvent in a modified belt-type high-pressure and high-temperature apparatus [23]. Samples of thickness ranging from 1 to $15 \mu \mathrm{m}$ were cleaved from h-BN single crystalline platelets. Optical transmittance and reflectance experiments in a wide spectral range from MIR to deep UV were performed in several homemade laboratory setups. Optical reflectance measurements in the MIR range (1800-7000 $\mathrm{cm}^{-1}$ ) were performed by means of Fourier transform infrared spectroscopy (FTIR) using an Interspectrum TEO-400 Michelson interferometer module and a noncommercial all-reflecting microscope optical bench [24]. For transmittance spectra in the near IR, visible, and UV range, halogen and deuterium lamps were used as sources, along with an all-reflecting optical bench, with long focal length concave mirrors, and a series of multichannel spectrometers covering a wavelength range from 1700 to $184 \mathrm{~nm}$. For transmission measurements at various incidence angles, the sample was mounted on a rotation stage equipped with an angle and position centering system. Normal incidence was checked by verifying that transmission spectra for a given angle were identical, irrespective of the rotation direction.

\section{RESULTS AND DISCUSSION}

Figure 1 (symbols) shows the refractive index spectrum $n_{\perp}(\sigma)$ of h-BN in the MIR range for polarization perpendicular to the $c$ axis, as obtained from the interference fringe pattern of the reflectance spectrum. The reflectance minima are given by the constructive interference condition $2 n_{\perp}(\sigma) d=K$, where $\sigma$ is the wave number, $d$ is the sample thickness, and $K$ is the interference order. The interference order and the sample thickness can be self-consistently determined by matching the interference minima to the refractive index determined from the dielectric function of a polar mode, which is given by the well-known expression

$$
\varepsilon^{\perp}(\sigma)=\varepsilon_{\infty}^{\perp}+\left(\varepsilon_{0}^{\perp}-\varepsilon_{\infty}^{\perp}\right) \frac{\sigma_{\mathrm{TO} \perp}^{2}}{\sigma_{\mathrm{TO} \perp}^{2}-\sigma^{2}-i \Gamma_{\perp} \sigma} .
$$

Here $\varepsilon_{0}^{\perp}$ and $\varepsilon_{\infty}^{\perp}$ are, respectively, the static and electronic contributions to the dielectric constant for light polarization

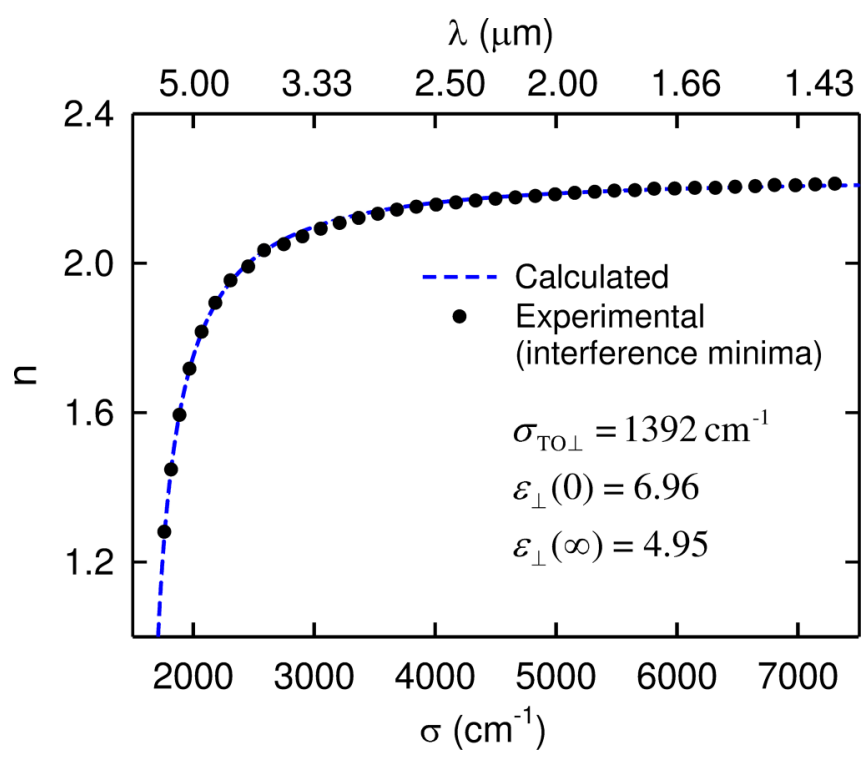

FIG. 1. Experimental values of the refractive index for polarization perpendicular to the $c$ axis in the MIR range as determined from the constructive interference condition at the interference minima of the reflectance spectrum of a $13.5-\mu \mathrm{m}$-thick h-BN sample (filled dots). The refractive index calculated using Eq. (1) with the parameter values given in the figure is plotted as a blue dashed line.

perpendicular to the $c$ axis, $\sigma_{\mathrm{TO} \perp}$ is the frequency of the TO phonon polarized perpendicular to the $c$ axis ( $E_{1 u}$ symmetry), and $\Gamma_{\perp}$ is its damping parameter. The best fit (blue dashed line in Fig. 1) was obtained for the values $\sigma_{\mathrm{TO} \perp}=1392 \mathrm{~cm}^{-1}$, $\varepsilon_{0}^{\perp}=6.96, \varepsilon_{\infty}^{\perp}=4.95, \Gamma_{\perp}=3 \mathrm{~cm}^{-1}$, and a sample thickness $d=13.5 \mu \mathrm{m}$. The $\sigma_{\mathrm{TO} \perp}$ value is in good agreement with experimental and theoretical values of the $E_{1 u}(\mathrm{TO})$ frequency previously reported [15,25].

Once the refractive index dispersion $n_{\perp}(\sigma)$ is established in the MIR range, its frequency dependence in the NIR, visible, and UV range can be determined from the transmittance interference fringe pattern of thinner samples (3.4 and $1.7 \mu \mathrm{m}$ thick). The interference order and sample thickness are evaluated by nonlinear fits in the MIR range and then the refractive index is obtained from the constructive interference condition. Figure 2 shows the refractive index spectrum of h-BN, for polarization perpendicular to the $c$ axis and frequencies above the restrahlen band. Symbols correspond to the experimental values determined by the above method, whereas dashed and solid lines are the results of several simple physical models that were applied to quantitatively account for the refractive index dispersion. The green line was calculated using Eq. (1) by assuming a constant electronic contribution to the dielectric function. The fit of this simple model illustrates the fact that below $7000 \mathrm{~cm}^{-1}$ the refractive index dispersion is practically determined by polar phonons.

The blue curve was obtained by assuming that the electronic contribution to the refractive index dispersion obeys a simple Phillips-van Vechten model [26,27]. This model assumes that all valence-to-conduction band transitions that contribute to the material electronic dielectric function can be described by a single transition at an average frequency which is known as Penn gap [28]. Under this assumption, the electronic dielectric 


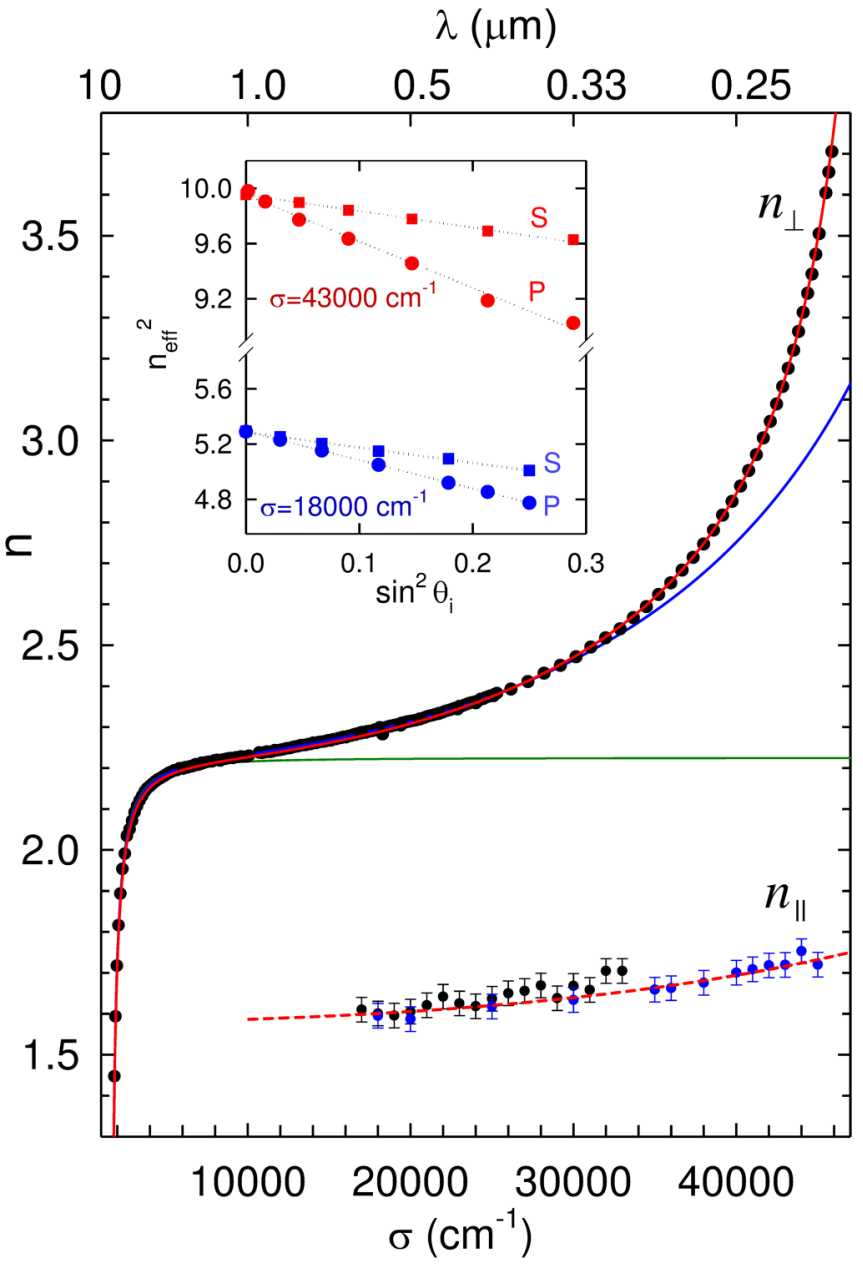

FIG. 2. Experimental values of h-BN refractive index for polarization perpendicular and parallel to the $c$ axis obtained from the interference pattern of the transmission spectra (filled dots). For $n_{\perp}$, the solid green line was calculated using Eq. (1) assuming a constant electronic dielectric constant. The blue line additionally includes a simple Phillips-van Vechten model [Eq. (2)] and the red line is obtained by considering also an additional excitonic term [Eq. (3)]. For $n_{\|}$, the dashed red line was obtained by fitting Eq. (2) to the data. Inset: Squared effective refractive index at $\sigma=18000 \mathrm{~cm}^{-1}$ (blue) and $\sigma=43000 \mathrm{~cm}^{-1}$ (red) as a function of the squared sinus of the incidence angle for $s$ - (filled squares) and $p$-polarized (filled dots) light. The slope of the linear fit (dotted lines) to the $p$-polarization data allows $n_{\|}$to be accurately determined.

function is given by

$$
\varepsilon_{\infty}^{\perp}(\sigma)=1+\frac{\sigma_{P 0 \perp}^{2}}{\sigma_{0 \perp}^{2}-\sigma^{2}},
$$

where $\sigma_{0 \perp}$ and $\sigma_{P 0 \perp}$ are, respectively, the Penn gap frequency and an average plasma frequency of valence electrons involved in allowed transitions for polarization perpendicular to the $c$ axis. The best fit to the experimental results was obtained with a Penn gap of $63000 \mathrm{~cm}^{-1}(7.8 \mathrm{eV})$ and a plasma frequency of $124800 \mathrm{~cm}^{-1}(15.5 \mathrm{eV})$. This model accounts for the refractive index dispersion up to $30000 \mathrm{~cm}^{-1}$, but obviously cannot explain the steep increase of the refractive index for photon energies approaching the fundamental gap of
$\mathrm{h}-\mathrm{BN}$. This discrepancy is a clear indication that, in spite of the well-established indirect character of h-BN [8], there must be an allowed transition at an energy slightly above the indirect band gap whose contribution to the refractive index cannot be neglected.

While the direct gap contribution to the refractive index can be accurately reproduced in many semiconductors by assuming a steplike absorption onset at the band gap energy [29,30], this model does not seem to fit the electronic structure of h-BN. In agreement with $a b$ initio band-structure calculations [7], which suggest strong excitonic effects in the dielectric function of h-BN, an additional Lorentzian term corresponding to an excitonic transition with central frequency $\sigma_{1 \perp}$, damping parameter $\Gamma_{1 \perp}$, and effective plasma frequency $\sigma_{\mathrm{P} 1 \perp}$ was included in Eq. (2) in order to obtain a better fit to the experimental refractive index dispersion $n_{\perp}(\sigma)$. Then, the dielectric function for polarization perpendicular to the $c$ axis is given by

$$
\varepsilon_{\infty}^{\perp}(\sigma)=1+\frac{\sigma_{P 0 \perp}^{2}}{\sigma_{0 \perp}^{2}-\sigma^{2}}+\frac{\sigma_{P 1 \perp}^{2}\left(\sigma_{1 \perp}^{2}-\sigma^{2}\right)}{\left(\sigma_{1 \perp}^{2}-\sigma^{2}\right)^{2}+\Gamma_{1 \perp}^{2} \sigma^{2}} .
$$

Given that the explored range of wave numbers showing interference does not include the refractive index maximum associated with the excitonic term in Eq. (3), a degree of uncertainty in the determination of $\sigma_{1 \perp}$ exists, and good fits to the experimental data can be obtained with different sets of parameters. We considered as a reference an experimental study of the dielectric constant in pyrolytic h$\mathrm{BN}$ [31], which revealed an intense peak at $6.1 \mathrm{eV}$ in its imaginary part. Then, the best fit to the experimental points (red line in Fig. 2) was obtained with $\sigma_{1 \perp}=49200 \mathrm{~cm}^{-1}$ $(6.1 \mathrm{eV}), \sigma_{P 1 \perp}=53200 \pm 800 \mathrm{~cm}^{-1}(6.60 \pm 0.10 \mathrm{eV})$, and $\Gamma_{1 \perp}=1560 \pm 270 \mathrm{~cm}^{-1}(0.193 \pm 0.033 \mathrm{eV})$. Obviously the introduction of the additional excitonic term implies a shift of the Penn gap contribution to higher energy. The new values of the Penn gap parameters we find from Eq. (3) are $\sigma_{0 \perp}=75100 \pm 1300 \mathrm{~cm}^{-1}(9.31 \pm 0.16 \mathrm{eV})$ and $\sigma_{P 0 \perp}=$ $124100 \pm 1400 \mathrm{~cm}^{-1}(15.39 \pm 0.17 \mathrm{eV})$. The excitonic lines contributing to the strong peak in the imaginary part of the dielectric constant were identified as originating from $\pi \rightarrow \pi^{*}$ interband transitions mainly with $\mathbf{k}$ points parallel to the $c$ axis in the direction $H K$ [7,31]. It should be stressed that because these excitonic lines involve bands with the same parity with respect to the mirror plane within each layer, they are only active for in-plane polarization.

The refractive index dispersion of $\mathrm{h}-\mathrm{BN}$ for light polarization perpendicular to the $c$ axis $\left(n_{\perp}\right)$ and parallel to the $c$ axis $\left(n_{\|}\right)$was determined from the interference fringe pattern of transmission spectra taken at different incidence angles $\theta_{i}$ by rotating the sample about an axis perpendicular to the optical axis of the setup. Since the samples were cleaved with faces perpendicular to the $c$ axis, for light polarization perpendicular to the incidence plane ( $s$ polarization) only $n_{\perp}$ appears in the light propagation equations, as only the ordinary ray propagates in the sample. Then, the interference maxima in the transmission spectrum are given by the constructive interference condition [32]

$$
\frac{2 n_{\perp} d}{\lambda} \sqrt{1-\frac{\sin ^{2} \theta_{i}}{n_{\perp}^{2}}}=\frac{2 d}{\lambda} n_{\mathrm{eff}, s}\left(\theta_{i}\right)=K,
$$


where we have defined the effective index $n_{\mathrm{eff}, s}$ as

$$
n_{\mathrm{eff}, s}=n_{\perp} \sqrt{1-\frac{\sin ^{2} \theta_{i}}{n_{\perp}^{2}}} .
$$

Note that $n_{\mathrm{eff}, s}$ is a purely instrumental parameter for the determination of the actual refractive index $n_{\perp}$. For $p$-polarized light, both $n_{\perp}$ and $n_{\|}$affect the light propagation inside the sample, and the transmission spectrum at a given incidence angle exhibits interference maxima at wavelengths determined by the following equation [32]:

$$
\frac{2 n_{\perp} d}{\lambda} \sqrt{1-\frac{\sin ^{2} \theta_{i}}{n_{\|}^{2}}}=\frac{2 d}{\lambda} n_{\mathrm{eff}, p}\left(\theta_{i}\right)=K .
$$

As in the case of $s$-polarized light, an effective index $n_{\text {eff, } p}$ for each incidence angle can be defined as

$$
n_{\mathrm{eff}, p}=n_{\perp} \sqrt{1-\frac{\sin ^{2} \theta_{i}}{n_{\|}^{2}}} .
$$

Transmission spectra recorded on the 3.4- $\mu \mathrm{m}$-thick sample for $s$ and $p$ polarizations for a number of incidence angles are shown in the Supplemental Material [33]. From the interference patterns, with the interference order $K$ and the sample thickness determined from the analysis of the MIR reflectivity, the effective index dispersion for $s$ - and $p$-polarized light can be obtained. Plots of the effective index dispersion are given in the Supplemental Material [33].

According to Eqs. (5) and (7), plots of the square of the effective indices versus $\sin ^{2} \theta_{i}$ at a given wave number should yield straight lines for both polarization conditions. For $n_{\mathrm{eff}, s}$ the slope is -1 , whereas for $n_{\mathrm{eff}, p}$ the slope equals the squared anisotropy ratio $\left(n_{\perp} / n_{\|}\right)^{2}$, from which the value of $n_{\|}$can be accurately determined. The inset of Fig. 2 illustrates this method for two wave numbers. While the slope of $n_{\mathrm{eff}, s}$ is found to be $1 \pm 0.05$ for both wave numbers, the slope of $n_{\mathrm{eff}, p}$ varies from 2 in the visible range to 4 in the UV range. It should be stressed that the main source of error associated with this method comes from the error in the incidence angle.

Figure 2 also shows the values of the refractive index of h-BN for polarization parallel to the $c$ axis. The dashed red line is a fit of a simple model equivalent to Eq. (2) with the parameters for light polarization parallel to the $c$ axis. The best fit is obtained for $\sigma_{0 \|}=90000 \mathrm{~cm}^{-1}(11.2 \mathrm{eV})$ and $\sigma_{P 0 \|}=$ $110000 \mathrm{~cm}^{-1}(13.7 \mathrm{eV})$, which yields an electronic dielectric constant $\varepsilon_{\infty}^{\|}=2.86$. This is in excellent agreement with the dielectric constant values predicted by $a b$ initio calculations $[7,16]$.

Even in the visible-NIR range $h-B N$ exhibits a giant negative birefringence $\left(\Delta n=n_{e}-n_{o} \sim-0.7\right)$ which is much higher in absolute value than the birefringence found, for instance, in $\mathrm{YVO}_{4}(\Delta n=0.21$ at $633 \mathrm{~nm})$, a standard example of a birefringent crystal that is widely used in laser devices and optical components [34]. These results are in stark contrast with previously published infrared reflectivity measurements on pyrolytic h-BN [15], which suggested a much smaller anisotropy ( $\Delta n \sim-0.2$ ). As pointed out above, the origin of this discrepancy is most likely related to the relatively poor crystalline quality of the h-BN samples available at the time of Ref. [15]. The optical anisotropy of h-BN increases markedly

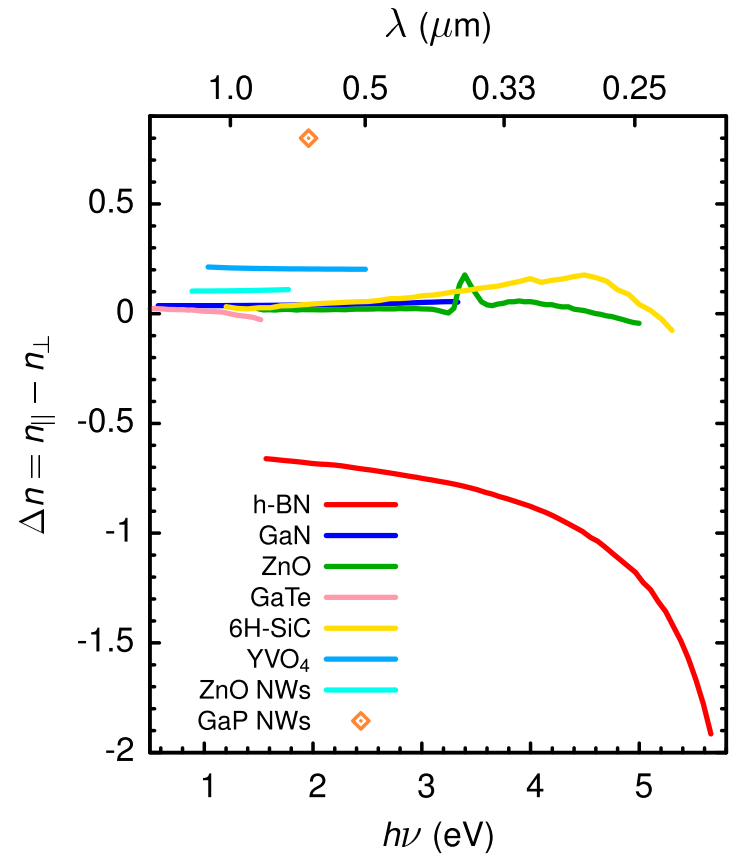

FIG. 3. Birefringence of h-BN in the NIR-UV range compared with the birefringence of several representative uniaxial semiconductors reported in the literature. The giant birefringence of $\mathrm{GaP}$ and $\mathrm{ZnO}$ ensemble nanowires is also displayed for further comparison.

in the UV range as the wave number approaches the band gap because of the dramatic increase of the dielectric function for polarization perpendicular to the $c$ axis. Such a vast increase in $\varepsilon_{\infty}^{\perp}(\sigma)(140 \%$ from 3.2 to $5.7 \mathrm{eV})$, which was modeled by including a Lorentzian excitonic term in Eq. (3), arises from the huge excitonic effects taking place in h-BN. As a consequence of the layered two-dimensional character of the h-BN crystal, the charge density inhomogeneity is large along the $c$ axis and the excitons are tightly confined within the layers. Low-lying excitons stem from interband transitions between conduction and valence bands of the same parity with respect to the mirror plane within each layer and they exhibit a large oscillator strength [7,31]. Parity selection rules allow these transitions only for polarization perpendicular to the $c$ axis. Therefore they contribute to $\varepsilon_{\perp}(\sigma)$ but not to $\varepsilon_{\|}(\sigma)$, and as a result, the giant birefringence of $h-B N$ shows a sizable increase in the UV range.

In Fig. 3 we compare the birefringence of h-BN measured over the NIR-UV range in this work with birefringence data on a selected group of birefringent semiconductor crystals available in the literature. In the visible range, the $\mathrm{h}-\mathrm{BN}$ birefringence in absolute value is more than one order of magnitude higher than in other uniaxial semiconductors such as wurtzite-type $\mathrm{GaN}$ [35] and $\mathrm{ZnO}$ [36] or $6 \mathrm{H}-\mathrm{SiC}$ [37]. The birefringence in other layered semiconductors such as GaTe [38] is also more than one order of magnitude lower. Highly birefringent photonic metamaterials based on the artificial birefringence of aligned nanowires are being developed for optical applications. For oblique-aligned $\mathrm{ZnO}$ nanowires, the birefringence is almost one order of magnitude higher than that of bulk $\mathrm{ZnO}$ [39], but still much less than the one measured in h-BN. Only the record values of $\Delta n=0.8$ at 
an optical wavelength of $633 \mathrm{~nm}$ achieved in $\mathrm{GaP}$ ensemble nanowires with very large filling fractions [20] can compare with the optical anisotropy observed at this wavelength in $\mathrm{h}-\mathrm{BN}$. And more strikingly, the birefringence of h-BN displays a huge increase in absolute value for increasing wave numbers in the UV range. This behavior is not observed in other uniaxial semiconductors or layered compounds, and is linked to the unique electronic structure of the layered h-BN crystal which underpins the strong electron-hole interaction and huge excitonic effects present in this material.

\section{CONCLUSIONS}

In-plane and out-of-plane refractive index dispersion of $\mathrm{h}$-BN has been determined over a wide energy range, from MIR up to UV energies slightly above the band gap, by analyzing the interference patterns in transmission measurements performed at different incidence angles and with polarization parallel and perpendicular to the incidence plane. Our results reveal an extreme variation of the ordinary ray refractive index $n_{\perp}$ from 1.5 in the MIR range up to 3.7 in the UV range close to the band gap. A simple Penn gap model fails to account for such a huge increase. The experimental results can be quantitatively explained by considering a strong excitonic peak contribution to the dielectric function for polarization perpendicular to the $c$ axis. This finding is in agreement with previous $a b$ initio calculations that indicate the presence of huge excitonic effects in layered h-BN and predict excitonic transitions with large oscillator strengths that are symmetry allowed only for polarization perpendicular to the $c$ axis. The refractive index for polarization parallel to the $c$ axis $\left(n_{\|}\right)$ is significantly lower than $n_{\perp}\left(n_{\|} \sim 1.6-1.7\right)$ and shows a much less marked increase as excitonic transitions are not allowed by the polarization selection rules. This results in a giant birefringence that steadily increases towards the UV range, where birefringence values as high as $|\Delta n| \sim 2$ have been measured. This is about seven times higher than the birefringence measured in calcite at the same wavelength [40]. Such a giant anisotropy has not been reported in other uniaxial semiconductors or layered compounds, nor even in engineered birefringent metamaterials. In the visible range, only high filling fraction $\mathrm{GaP}$ nanowire layers exhibit a comparable birefringence. Remarkably, in the UV range the birefringence in h-BN is sizably larger than in any other known material.

From the fits of the dielectric function model we obtain values of the electronic dielectric constants $\varepsilon_{\infty}^{\perp}=4.95$ and $\varepsilon_{\infty}^{\|}=2.86$. The dielectric constant we find for polarization parallel to the $c$ axis is much lower than previously reported experimental values, but it is in excellent agreement with theoretical calculations. Thus, our measurements reveal that h-BN exhibits a much stronger birefringence than previously thought and therefore pinpoint h-BN as a material of outstanding interest for optical applications that require huge birefringence components.

\section{ACKNOWLEDGMENTS}

This work has been supported by the Spanish MINECO/FEDER under Contracts No. MAT2015-71035-R and No. MAT2016-75586-C4-1-P. B.G. acknowledges the Russian Megagrant program (Ioffe Institute of RAS, Contract No. 14.W03.31.0011). T.T. acknowledges support from the Elemental Strategy Initiative conducted by the MEXT, Japan and JSPS KAKENHI Grant No. JP15K21722.
[1] K. Watanabe, T. Taniguchi, and H. Kanda, Nat. Mater. 3, 404 (2004).

[2] B. Huang, X. K. Cao, H. X. Jiang, J. Y. Lin, and S.-H. Wei, Phys. Rev. B 86, 155202 (2012).

[3] Y. Kubota, K. Watanabe, O. Tsuda, and T. Taniguchi, Science 317, 932 (2007)

[4] K. Watanabe, T. Taniguchi, T. Niiyama, K. Miya, and M. Taniguchi, Nat. Photon. 3, 591 (2009).

[5] K. Watanabe and T. Taniguchi, Phys. Rev. B 79, 193104 (2009).

[6] X. Blase, A. Rubio, S. G. Louie, and M. L. Cohen, Phys. Rev. B 51, 6868 (1995).

[7] B. Arnaud, S. Lebègue, P. Rabiller, and M. Alouani, Phys. Rev. Lett. 96, 026402 (2006).

[8] G. Cassabois, P. Valvin, and B. Gil, Nat. Photon. 10, 262 (2016).

[9] A. Mishchenko, J. S. Tu, Y. Cao, R. V. Gorbachev, J. R. Wallbank, M. T. Greenaway, V. E. Morozov, S. V. Morozov, M. J. Zhu, S. L. Wong, F. Withers, C. R. Woods, Y.-J. Kim, K. Watanabe, T. Taniguchi, E. E. Vdovin, O. Makarovsky, T. M. Fromhold, V. I. Fal'ko, A. K. Geim, L. Eaves, and K. S. Novoselov, Nat. Nanotechnol. 9, 808 (2014).

[10] Q. Li, M. Liu, Y. Zhang, and Z. Liu, Small 12, 32 (2016).

[11] L. Wang, I. Meric, P. Y. Huang, Q. Gao, Y. Gao, H. Tran, T. Taniguchi, K. Watanabe, L. M. Campos, D. A. Muller, J. Guo,
P. Kim, J. Hone, K. L. Shepard, and C. R. Dean, Science 342, 614 (2013).

[12] M. P. Levendorf, C.-J. Kim, L. Brown, P. Y. Huang, R. W. Havener, D. A. Muller, and J. Park, Nature (London) 488, 627 (2012).

[13] A. Maity, T. C. Doan, J. Li, J. Y. Lin, and H. X. Jiang, Appl. Phys. Lett. 109, 072101 (2016).

[14] Y. Panayiotatos, S. Logothetidis, A. Laskarakis, A. Zervopoulou, and M. Gioti, Diamond Relat. Mater. 11, 1281 (2002).

[15] R. Geick, C. H. Perry, and G. Rupprecht, Phys. Rev. 146, 543 (1966).

[16] N. Ohba, K. Miwa, N. Nagasako, and A. Fukumoto, Phys. Rev. B 63, 115207 (2001).

[17] M. J. A. de Dood, W. T. M. Irvine, and D. Bouwmeester, Phys. Rev. Lett. 93, 040504 (2004).

[18] H. Pettersson, J. Trägårdh, A. I. Persson, L. Landin, D. Hessman, and L. Samuelson, Nano Lett. 6, 229 (2006).

[19] M. V. Kotlyar, L. Bolla, M. Midrio, L. O'Faolain, and T. F. Krauss, Opt. Express 13, 5040 (2005).

[20] O. L. Muskens, M. T. Borgström, E. P. A. M. Bakkers, and J. Gómez Rivas, Appl. Phys. Lett. 89, 233117 (2006).

[21] Y. Terada, K. Shimamura, and T. Fukuda, J. Alloys Compd. 275-277, 697 (1998). 
[22] T. Low, A. Chaves, J. D. Caldwell, A. Kumar, N. X. Fang, P. Avouris, T. F. Heinz, F. Guinea, L. Martin-Moreno, and F. Koppens, Nat. Mater. 16, 182 (2016).

[23] T. Taniguchi and K. Watanabe, J. Cryst. Growth 303, 525 (2007).

[24] V. Panchal, A. Segura, and J. Pellicer-Porres, High Pressure Res. 31, 445 (2011).

[25] R. Cuscó, B. Gil, G. Cassabois, and L. Artús, Phys. Rev. B 94, 155435 (2016).

[26] J. C. Phillips, Rev. Mod. Phys. 42, 317 (1970).

[27] J. A. Van Vechten, Phys. Rev. 182, 891 (1969).

[28] D. R. Penn, Phys. Rev. 128, 2093 (1962).

[29] S. Adachi, Phys. Rev. B 35, 7454 (1987).

[30] A. R. Goñi, K. Syassen, and M. Cardona, Phys. Rev. B 41, 10104 (1990).

[31] D. M. Hoffman, G. L. Doll, and P. C. Eklund, Phys. Rev. B 30, 6051 (1984).
[32] H. Schopper, Z. Phys. 132, 146 (1952).

[33] See Supplemental Material at http://link.aps.org/supplemental/ 10.1103/PhysRevMaterials.2.024001 for interference patterns in transmission spectra recorded at different incidence angles and effective index dispersion.

[34] See for instance: www.optoscience.com.

[35] S. Shokhovets, M. Himmerlich, L. Kirste, J. H. Leach, and S. Krischok, Appl. Phys. Lett. 107, 092104 (2015).

[36] H. Yoshikawa and S. Adachi, Jpn. J. Appl. Phys. 36, 6237 (1997).

[37] S. Ninomiya and S. Adachi, Jpn. J. Appl. Phys. 33, 2479 (1994).

[38] J. F. Sánchez-Royo, A. Segura, and V. Muñoz, Phys. Status Solidi A 151, 257 (1995).

[39] C.-Y. Chen, J.-H. Huang, K.-Y. Lai, Y.-J. Jen, C.-P. Liu, and J.-H. He, Opt. Express 20, 2015 (2012).

[40] G. Ghosh, Opt. Commun. 163, 95 (1999). 